\title{
VERY LONG BASELINE INTERFEROMETRY OBSERVATIONS USING THE TRACKING AND DATA RELAY SATELLITE AS AN ORBITING RADIO TELESCOPE
}

\author{
R. P. Linfield ${ }^{1}$, G. S. Levy ${ }^{1}$, J. S. Ulvestad ${ }^{1}$, C. D. Edwards ${ }^{1}$, J. F. \\ Jordan, Jr. ${ }^{1}$, S. J. DiNardo ${ }^{1}$, C. S. Christensen ${ }^{1}$, R. A. Preston ${ }^{1}$, \\ L. J. Skjerve ${ }^{1}$, L. R. Stavert ${ }^{1}$, B. F. Burke ${ }^{2}$, A. R. Whitney ${ }^{3}$, R. \\ J. Cappallo ${ }^{3}$, A. E. E. Rogers ${ }^{3}$, K. B. Blaney ${ }^{4}$, M. J. Maher ${ }^{5}$, C. \\ H. Ottenhoff ${ }^{6}$, D. L. Jauncey ${ }^{7}$, J. E. Reynolds ${ }^{8}$, T. Nishimura ${ }^{9}$, \\ T. Hayashi ${ }^{9}$, T. Takano ${ }^{9}$, T. Yamada ${ }^{9}$, H. Hirabayashi ${ }^{10}$, M.

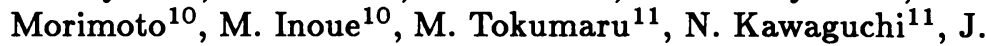 \\ Amagai $^{11}$ \\ ${ }^{1}$ Jet Propulsion Laboratory, Pasadena, CA 91109 \\ ${ }^{2}$ Massachusetts Institute of Technology, Cambridge, MA 02139 \\ ${ }^{3}$ Haystack Observatory, Westford, MA 01886 \\ ${ }^{4}$ Goddard Space Flight Center, Greenbelt, MD 20771 \\ ${ }^{5}$ Bendix Field Engineering Corporation, Seabrook, MD 20706 \\ ${ }^{6}$ TRW Space and Technology Group, Las Cruces, NM 88004 \\ ${ }^{7}$ Division of Radiophysics, Commonwealth Scientific and Industrial \\ Research Organization, Canberra, ACT 2601, Australia \\ ${ }^{8}$ Mount Stromlo Observatory, Canberra, ACT 2606, Australia \\ ${ }^{9}$ Institute for Space and Astronautical Science, Tokyo, 153, Japan \\ ${ }^{10}$ Nobeyama Radio Observatory, Nobeyama, 384-13, Japan \\ ${ }^{11}$ Radio Research Laboratory, Kashima, 314, Japan
}

ABSTRACT. An antenna in geostationary orbit was used for VLBI observations at $2.3 \mathrm{GHz}$, in combination with ground antennas in Australia and Japan. 23 of the 25 observed sources were detected on orbiter-ground baselines, with baseline lengths as large as 2.15 earth diameters. Brightness temperatures between $10^{12} \mathrm{~K}$ and $4 \times 10^{12} \mathrm{~K}$ were measured for 10 sources.

\section{OBSERVATIONS}

VLBI observations using a $4.9 \mathrm{~m}$ diameter antenna of the Tracking Data and Relay Satellite System were first performed in July and August 1986 (Levy et al., 1986). In January 1987, additional observations were made, again using $64 \mathrm{~m}$ diameter ground antennas in Tidbinbilla, Australia and Usuda, Japan. The observing frequency was $2.3 \mathrm{GHz}$. 25 sources were observed, with a total observing time of 20 hours. The sources ranged in declination from $0^{\circ}$ to $-30^{\circ}$, and were observed at spacecraft hour angles of $180^{\circ} \pm 22^{\circ}$ (i.e. nearly towards the earth). 


\section{DATA REDUCTION}

The data were correlated at Haystack, using the Mk IIIA correlator. Due to software limitations, it has not yet been possible to obtain closure phase values. The coherence fell significantly at short integration times, and then dropped more slowly. Measured values were 0.94 at 30 s., 0.86 at 100 s., 0.78 at 200 s., and 0.68 at $600 \mathrm{~s}$. These are mean values; the standard deviations were $3 \%$ at 30 s., $9 \%$ at 100 s., $12 \%$ at 200 s., and $19 \%$ at 600 s. The reason for such a time-variable coherence is not known, but instabilities in the spacecraft electronics are suspected.

\section{RESULTS}

Of the 25 sources which were observed, 23 were detected, with data from one other lost due to equipment problems. The maximum baseline for each source depended primarily on declination, and ranged from 0.94 to 2.15 earth diameters. Visibilities on the maximum baseline for each source ranged from 0.11 to 0.68 . The most compact source was $1519-273$, with a visibility of $0.68(1.1 \mathrm{Jy}$ correlated flux) on a baseline of 2.02 earth diameters. With a fitted size of 0.33 milliarcseconds, the brightness temperature is $3.4 \times 10^{12} \mathrm{~K}(1+z)$ (the redshift for this source is not known). Other sources with very high brightness temperatures were: $0336-019\left(2.4 \times 10^{12} \mathrm{~K}\right), 0420-014\left(3.3 \times 10^{12} \mathrm{~K}\right), 1921-293$ $\left(3.8 \times 10^{12} \mathrm{~K}\right)$, and $2216-038\left(3.4 \times 10^{12} \mathrm{~K}\right)$. The errors on these values range from $10 \%$ to $35 \%$.

\section{DISCUSSION}

Baseline lengths greater than 1 earth diameter are necessary to measure the structure of numerous compact radio sources. Two (1519-273 and 1921-293) of the three sources observed on baselines greater than 2 earth diameters displayed correlated flux greater than $1 \mathrm{Jy}$ on those baselines. However, none of the sources were completely unresolved. Baselines of 1-3 earth diameters, available only with orbiting VLBI, promise to reveal a great deal about the structure of compact radio sources.

The measured brightness temperatures for 10 sources exceed $10^{12} \mathrm{~K}$. If incoherent synchrotron radiation is the emission mechanism in these sources, the Inverse Compton process will limit the brightness temperature to $<10^{12} \mathrm{~K}$ in the source rest frame. Bulk relativistic motion in the direction towards us, with a Doppler factor of at least 1.5-2, is required to explain the observations. This provides additional evidence that many (if not most) compact radio sources are significantly Doppler boosted in flux.

\section{REFERENCES}

Levy, G. S. et al. 1986, Science, 234, 187. 